\title{
Efectividad de Estrategias de Prevención de Violencia Escolar: La Experiencia del Programa Recoleta en Buena
}

\section{Effectiveness of Prevention Strategies for School Violence: The Experience of the Program Recoleta en Buena}

\author{
Jorge Varela \\ Fundación Paz Ciudadana
}

\begin{abstract}
Se describen los resultados de la evaluación de efectividad de un programa destinado a disminuir la violencia escolar en 4 establecimientos municipales de la comuna de Recoleta, perteneciente a la Región Metropolitana de Chile. Se usó un diseño pre-experimental de preprueba-postprueba con un solo grupo. Fueron evaluados 677 estudiantes en 2006 y 553 en 2008 , de $5^{\circ}$ básico a $4^{\circ}$ medio. Se consideraron 8 factores de violencia, según la percepción de testigos, víctimas, agresores y del clima escolar. Hubo una disminución de la violencia entre ambos años en los factores Observador y Víctima de Violencia Entre Pares, Observador de Violencia Antisocial, Observador de Violencia de Alumnos a Adultos y de Profesores a Alumnos y Victimario de Violencia a Compañeros. Al comparar los mismos niveles en ambos años, solo disminuyen Observador y Víctima de Violencia de Profesores a Alumnos y Observador de Violencia de Alumnos a Adultos. Los resultados muestran efectividad del programa en materias promocionales y preventivas, pero no en conductas más graves de violencia. Se destaca la importancia de avanzar en la evaluación de programas de violencia escolar.
\end{abstract}

Palabras clave: violencia escolar, programa de intervención, evaluación, Chile

\begin{abstract}
The results of the evaluation of the effectiveness of a program aimed to reduce school violence in 4 public schools of Recoleta, in the Metropolitan Region of Chile, are described. A pretest-posttest pre-experimental design with a single group was used. In 2006 and 2088, 677 and 553 students from $5^{\text {th }}$ to $12^{\text {th }}$ grade, respectively, were evaluated. Eight factors of violence, as perceived by witnesses, victims, aggressors, and the school climate, were assessed. There was a decrease in violence between both years in the factors Observer and Victim of Peer Violence, Observer of Antisocial Violence, Observer of Violence From Students to Adults and From Teachers to Students, and Perpetrator of Peer Violence. When the same grades were followed, only Observer and Victim of Violence From Teachers to Students and Observer of Violence From Students to Teachers show a reduction. The results show effectiveness of the program in preventive and promotional matters, but not in more serious violent behaviors. The importance to progress in the evaluation of school violence programs is highlighted.
\end{abstract}

Keywords: school violence, intervention program, evaluation, Chile

En el presente artículo se reportan los resultados de la efectividad de un programa de prevención, llamado Recoleta en Buena, en la reducción de violencia escolar. Se sabe que la violencia escolar, fenómeno complejo y multicausal, incide en la convivencia escolar, dificulta el aprendizaje y genera un daño en las víctimas, victimarios y en la comunidad escolar en su conjunto (Arón \& Milicic, 1999, 2000; Casassus, 2003). La investigación en Chile que ha abordado este fenómeno (e.g., Contador, 2001; García \& Madriaza 2005; Tamar, 2005) ha permitido conocer más sobre sus diferentes manifestaciones, pero todavía no hay estudios que se hayan centrado en adaptar y validar formas efectivas de prevenirlo, salvo algunos estudios pioneros en la materia (Arón, 2000; Pérez, Rodríguez, De la Barra \& Fernández, 2005).

Jorge Varela Torres, Fundación Paz Ciudadana, Santiago, Chile.

El artículo corresponde a la Tesis para optar al título de Magíster en Psicología Educacional de la Escuela de Psicología de la Pontificia Universidad Católica de Chile.

La correspondencia relativa a este artículo debe ser dirigida a Jorge Varela Torres, Fundación Paz Ciudadana, Valenzuela Castillo 1881, Providencia, Santiago, Chile. E-mail: jvarela@pazciudadana.cl 


\section{Violencia Escolar}

Tomando como referencia la definición que hace la Organización Mundial de la Salud (Krug, Dalhberg, Mercy, Zwi \& Lozano, 2003), la violencia escolar puede ser entendida como toda aquella acción incluida dentro del ámbito de influencia de la escuela que por acto directo u omisión, ya sea en grado de amenaza o efectivo, tiene la intencionalidad de dañar al otro (ya sea un individuo, grupo o comunidad) o que cause o tenga muchas probabilidades de causar lesiones, muerte o daños psicológicos, trastornos del desarrollo o privaciones.

En base a los modelos explicativos que propuso Rigby (2003, 2004, 2005) y asumiendo la complejidad del fenómeno, Berger y Lisboa (2008) plantean que el análisis del fenómeno puede hacerse en tres niveles: individual, grupal y sociocultural. El primero se focaliza en las variables individuales asociadas al fenómeno, considerando, incluso, el aporte de las variables genéticas. En el segundo nivel se plantea que la aparición del fenómeno se da cuando los/las niños/as empiezan a relacionarse y buscan dominar a otros en un encuentro con distintas posiciones de poder. Este fenómeno se da en términos de disputas y/o defensa de la posesión de objetos y/o bienes que son considerados valiosos en el contexto escolar, por ejemplo, el estatus social. En el último nivel se plantea que esto se daría en un contexto de diferencias de estatus, las que ocurren en función de algunos antecedentes históricos y culturales (raza, género, nivel socioeconómico, entre otros). Cada uno de estos niveles, a pesar de contar con evidencia empírica de su validez, por sí solo no permite dar cuenta de toda la complejidad del fenómeno. Por lo mismo, es necesario asumir una mirada holística, desde una perspectiva ecológica del desarrollo humano (Bronfenbrenner, 1987), que considere elementos individuales, grupales y socioculturales.

\section{La Violencia Escolar y sus Implicancias Para la Intervención}

La violencia escolar puede ser entendida como una función de factores en distintos sistemas del colegio (Berger, Karimpour \& Rodkin, 2008; Espelage \& Swearer, 2003). Este enfoque permite tener una mejor comprensión del fenómeno y, al mismo tiempo, líneas de intervención para prevenirlo. En este sentido, desde el nivel individual se debe considerar el apoyo a los estudiantes que actualmente son victimarios y víctimas, el uso de estrategias de afrontamiento y romper con la cultura del silencio frente a las situaciones de maltrato. Por otro lado, se sugiere, además, promover el desarrollo de habilidades sociales (inteligencia emocional, resolución alternativa de problemas, desarrollo moral, por ejemplo) y ayudar a entender la función que juega la violencia en los agresores. A nivel diádico, es necesario trabajar con la cultura de pares, las normas que se establecen en los grupos y sus respectivas creencias, por ejemplo, que es "normal" molestar a un compañero que es diferente o menos popular. También requiere fortalecer los vínculos interpersonales (por ejemplo, fortalecer las comunidades de curso) y el vínculo con los profesores. Finalmente, desde lo institucional es importante impartir perfeccionamiento docente, debido a que son componentes centrales de cualquier programa, en fortalecer la convivencia y clima escolar, trabajar en las creencias que puedan existir respecto de la violencia y fortalecer una alianza familia-escuela (Berger et al., 2008; Berger \& Lisboa, 2008).

Asumir una perspectiva holística del fenómeno implica combinar en un programa de intervención estrategias de prevención y promoción. Cuando se desarrollan estrategias para controlar y erradicar las situaciones de victimización, en las que el foco está centrado en manifestaciones negativas como, por ejemplo, conducta agresiva, y se busca identificar los factores de riesgo y protectores asociados, se habla de prevención. Por otra parte, la promoción se centra en resultados positivos esperados en la comunidad escolar, donde todos se sientan protegidos y formando parte de ella (Berger et al., 2008; Berger \& Lisboa, 2008; Farrington, 1993; Orpinas \& Horne, 2006; Ortega, Del Rey \& Fernández, 2003).

Otros modelos de intervención con buenos resultados utilizan la lógica de prevención en salud, en los que se interviene en los niveles primario, secundario y terciario, lo cual se define según la evolución del fenómeno y acorde con las características de la manifestación de la violencia en los estudiantes. A nivel primario, se desarrollan estrategias universales e 
inespecíficas dirigidas hacia todos los estudiantes (por ejemplo, reforzar conductas positivas de los alumnos o el desarrollo curricular) (Chaux, 2005). A nivel secundario, se trabaja en forma grupal con algunos estudiantes que han sido identificados como en riesgo o que ya manifiestan conductas agresivas (por ejemplo, talleres de habilidades sociales) (Sugai \& Horner, 2006) y a nivel terciario, se realiza un apoyo focalizado e individual a estudiantes más necesitados, con graves problemas de violencia (Chaux, 2005; OSEP Center on Positive Behavioral Interventions et al., 2000; Rigby, Smith \& Pepler, 2004; Sprague \& Walker, 2000, 2005; Sugai, 2003, 2007; Sugai \& Horner, 2006; Turnbull et al., 2002).

Otros programas que han tenido buenos resultados son aquellos que promueven la capacitación docente respecto de cualquiera de los niveles antes descritos (Chaux, 2005). Ejemplos de intervención a nivel institucional son la enseñanza de normas de conducta o reglas claras contra el maltrato y monitoreo constante (Gottfredson, 1997; Gottfredson, Wilson \& Skroban, 2002; Olweus, 2004; Ortega et al., 2003; Rigby et al., 2004; Sugai, 2007; Welsh, 2007). El trabajo requiere establecer normas de conducta compartidas y aplicadas en forma homogénea, incorporando también a los padres y a los alumnos (Vanderschueren \& Lunecke, 2004). Otros aspectos generales destacados a considerar para la efectividad de estos programas son la duración y alcance de los programas (Gottfredson, 1997; Orpinas \& Horne, 2006) y contar con un plan de trabajo, un buen diagnóstico y una evaluación sostenida (Farrington, 1993; Ortega et al., 2003; Rigby et al., 2004).

Lo anterior sugiere que los programas con buenos resultados son aquellos que asumen abordajes en varios niveles: individual, curso, escuela, familia y ambiente físico; por ejemplo, contar con políticas contra la violencia escolar en la escuela, desarrollar currículos con énfasis en la prevención del racismo y los prejuicios, implementar técnicas específicas, como el desarrollo de la asertividad y el manejo de la ira, desarrollar reglas claras contra la victimización y vigilar y monitorear a los estudiantes y el espacio físico (Rigby et al., 2004).

\section{Evaluación de Programas de Prevención de Violencia Escolar}

Olweus (2004) realizó un meta-análisis de 400 programas de prevención de violencia y encontró que solamente 10 de ellos $(2,5 \%)$ lograron cumplir con algunos criterios mínimos de resultados, es decir, tener un efecto positivo en un grupo objetivo de estudiantes, que los efectos hayan durado al menos un año y contar con resultados positivos en alguna de las mediciones iniciales. En otro meta-análisis, que incluyó a 178 programas, los investigadores encontraron que las estrategias que tenían efectos positivos (medidas por el tamaño del efecto) eran aquellas que instalan una capacidad de innovación permanente, programas que clarificaban y comunicaban normas de conducta a los alumnos y programas centrados en un amplio rango de competencias sociales (autocontrol, manejo del estrés, por ejemplo), por un período largo de tiempo que permita ir reforzando esas habilidades (Gottfredson, 1997; Gottfredson et al., 2002).

A raíz de lo anterior, en el último tiempo se han establecido estándares para evaluar la efectividad de programas de prevención en general, por medio de la realización de meta-análisis más rigurosos. Un ejemplo de esto es la Escala de Maryland, la cual permite establecer un ranking de los estudios de evaluación de programas y, por ende, de sus resultados. Un mayor puntaje en la escala da cuenta de un mejor control de factores externos que puedan explicar los resultados evidenciados y, también, de los reales efectos del mismo, por ejemplo, por medio del tamaño del efecto (Przybylski, 2008; Sherman et al., 1997). Una evaluación que incorpore estos elementos permite señalar si un programa es efectivo; sin embargo, estos estándares de evaluación han sido incorporados en menor medida en la evaluación de programas de prevención en violencia escolar.

El objetivo del presente estudio fue analizar la efectividad de un programa de prevención de violencia escolar, con el fin de aportar información empírica sobre estrategias llevadas a cabo en Chile. La efectividad se entendió como el producto de la ecuación entre los resultados y los objetivos propuestos de un programa, el que permite interpretar el real logro del mismo. Por 
lo tanto, va más allá de la eficacia, ya que esta solo se refiere al grado de cumplimiento de los objetivos de un programa. Tampoco fue una evaluación de impacto, porque el diseño utilizado no logra controlar todos los efectos no atribuibles al programa (Cohen \& Franco, 1992).

En concreto, por medio del estudio se propuso evaluar de manera específica la efectividad de estrategias integrales y de prevención en: (a) la reducción de la victimización de violencia escolar por parte de estudiantes o profesores, (b) la reducción de alumnos que reportan ser agresores de violencia escolar hacia compañeros o docentes, (c) la reducción de la percepción de testigos de violencia escolar en el establecimiento entre compañeros y docentes y (d) la mejora en la percepción de las relaciones interpersonales al interior del establecimiento educacional.

Consistente con los resultados de investigaciones previas y la revisión de la literatura, se esperaba encontrar que, al cabo de la realización del programa en los cuatro establecimientos educacionales, los alumnos reportaran ser menos víctimas y victimarios de violencia escolar, ya sea respecto de sus pares como de sus profesores. A su vez, se esperaba encontrar en el rol de observador un menor reporte de violencia escolar, tanto de estudiantes como de profesores. Y, finalmente, se esperaba encontrar una mejor percepción de las relaciones interpersonales al interior del establecimiento educacional.

\section{El Programa Recoleta en Buena}

El programa evaluado en el presente estudio se denomina Recoleta en Buena, el cual nace en el año 2006 para prevenir e intervenir en episodios de violencia en las escuelas de la comuna del mismo nombre. En sus inicios esta línea de trabajo recibió el apoyo técnico de la Fundación Paz Ciudadana que, a través de Paz Educa (nombre original del programa), brindó la estructura base de la intervención. A través del tiempo y por medio del Departamento de Educación y la Dirección de Prevención Social, Mediación y Seguridad Ciudadana, el programa se fue adaptando a partir de las necesidades y recursos locales, configurando el modelo de trabajo que Recoleta en Buena llevó a cabo.

Dicho programa está basado en la experiencia de trabajo de Jeff Sprague en Estados Unidos, con el programa Positive Behavior Support (PBS), de la Universidad de Oregon. Se basa en identificar y organizar prácticas escolares efectivas, especialmente dirigidas a aquellos estudiantes que presentan problemas significativos de conducta (Sugai, 2007). Más que centrarse en la violencia, promueve una escuela saludable, centrada en el apoyo y desarrollo integral de sus estudiantes (Turnbull et al., 2002). Propone abordar la violencia desde cuatro subsistemas: nivel escolar, sala de clases, individual y familiar y comunidad. A nivel escolar, se traduce en promover normas de convivencia — expectativas de conducta—, desarrollo de habilidades sociales y una mejor supervisión del espacio físico del colegio, como la infraestructura y áreas comunes (patios, pasillos, comedor, entre otros). A nivel de sala de clases, se enseñan y refuerzan las normas de convivencia establecidas para todo el colegio de forma conjunta con toda la comunidad escolar. A nivel individual, se realizan intervenciones intensivas e individualizadas a alumnos/as con problemas de conducta más complejos. A nivel familiar y con la comunidad, se trabaja también con las normas de convivencia y en fortalecer alianzas de trabajo (OSEP Center on Positive Behavioral Interventions et al., 2000; Sprague \& Golly, 2005; Sugai \& Horner, 2006).

A su vez, es un programa de carácter preventivo, debido a que está dirigido a todos los estudiantes de la escuela y no solo a aquellos que presentan conductas más conflictivas, combinando intervenciones a nivel de prevención primaria, secundaria y terciaria. A nivel universal se trabaja enseñando habilidades sociales y se construyen y definen expectativas de conducta para los alumnos, las cuales son reforzadas y monitoreadas permanentemente. A nivel grupal se recomiendan acciones de enseñanza intensiva en habilidades sociales, estrategias de autocontrol, apoyo académico intensivo y tutorías. Finalmente, a nivel individual se traduce en un apoyo individual profesional de manejo y desarrollo de habilidades sociales, junto con la familia y la colaboración de múltiples servicios en red (OSEP Center on Positive Behavioral Interventions et al., 2000; Sprague \& Walker, 2000, 2005; Sugai, 2003, 2007; Sugai \& Horner, 2006; Turnbull et al., 2002). 
La metodología del PBS fue implementada anteriormente en Chile durante tres años en colegios de la comuna de Puente Alto, en forma piloto. Ahí fue posible adaptar algunos instrumentos y metodologías del programa, lo cual fue documentado, evaluado y sistematizado (Tijmes \& Varela, 2008, Noviembre; Varela, Farren \& Tijmes, 2010; Varela, Tijmes \& Sprague, 2009).

El programa Recoleta en Buena se implementó en cuatro establecimientos municipales de la comuna de Recoleta, los cuales fueron invitados a participar del programa a través de la municipalidad. Se contrató para ello a un psicólogo educacional, capacitado y supervisado durante dos años por la Fundación Paz Ciudadana. Además, el experto internacional Jeffrey Sprague viajó a Chile y trabajó con las cuatro escuelas durante su visita.

La primera etapa del programa consistió en crear un equipo de trabajo coordinador, con representantes de diferentes estamentos de los colegios. Este fue capacitado y supervisado por un profesional de la municipalidad durante los dos años del programa. En base a los niveles antes descritos, las acciones del programa fueron:

- A nivel de todo el colegio fueron definidas por toda la comunidad las normas de convivencia para los alumnos y un plan de enseñanza y refuerzo de las mismas a nivel institucional. Complementario a lo anterior, en cada colegio se realizaron mejoras físicas y situacionales, basadas en la metodología Prevención del crimen mediante el diseño ambiental (Crime Prevention Through Environmental Design; Schneider, Walker \& Sprague, 2000). Esto se ejecutó durante los dos años del programa.

- A nivel de salas de clases se trabajó con los cursos con más problemas de conducta y con sus profesores. Esto se implementó en el segundo año del programa.

- A nivel individual se desarrollaron tutorías con alumnos en riesgo, con el apoyo de las redes de salud de la comuna.

- A nivel familiar y comunitario se reforzaron las normas de conducta en las reuniones de apoderados y también se fortalecieron las redes sociales de cada escuela, con reuniones y coordinaciones con actores locales, por ejemplo, programas sociales que también trabajan en la comuna.

\section{Método}

El diseño del estudio fue de preprueba-postprueba con un solo grupo o colegio (Shadish, Cook \& Campbell, 2002) y pre-experimental, debido a que no se incluyó un grupo control (Hernández, Fernández \& Baptista, 1991).

\section{Participantes}

La muestra del estudio incluyó a estudiantes de cuatro establecimientos municipales de la comuna de Recoleta, que cursaban entre $5^{\circ}$ básico y $4^{\circ}$ medio. Los establecimientos educacionales participantes son de dependencia municipal, tres escuelas básicas y un liceo, catalogados por el Ministerio de Educación como de nivel socioeconómico medio-bajo, con un índice de vulnerabilidad escolar (IVE) promedio de 43\% en el año 2006. Esto quiere decir que los apoderados han declarado tener entre 8 y 10 años de estudio, un ingreso del hogar que varía entre $\$ 122.501$ y $\$ 195.000$ (entre 240 y 380 dólares) y los estudiantes se encuentran en condición de vulnerabilidad social en un rango de 40 a $60 \%$.

Participaron en la evaluación todos los alumnos que se encontraban presentes en el colegio en el día de la medición. De esta forma, se aplicó un cuestionario a 677 alumnos/as en el año 2006 y 553 alumnos/as en el año 2008 en los mismos cuatro colegios del estudio. En la primera medición (2006) el porcentaje de hombres fue 58\% y la media de edad fue 13,5 años (con un rango de 10 a 20 años de edad y una desviación típica de 2,2 años). En la segunda medición (2008) el porcentaje de hombres fue 57\% y la media de edad fue 13,5 años (con un rango de 10 a 19 años de edad y una desviación típica de 2,1 años). 


\section{Instrumento}

El instrumento utilizado para medir violencia escolar fue un cuestionario creado el año 2005 por Fundación Paz Ciudadana, usando como base cuestionarios elaborados en Europa y Estados Unidos (Ajenjo \& Bas, 2005; Araos \& Correa, 2004). Contiene 69 preguntas con alternativas de respuesta cerradas tipo Likert que miden relaciones interpersonales, ser víctima, victimario u observador de violencia escolar, según tipo y frecuencia, durante el año escolar.

Las preguntas que miden relaciones interpersonales (siete en total) tienen alternativas de respuesta que van desde Muy buena hasta Muy mala. Para medir la calidad de la relación se utilizó una escala tipo Likert de 1 a 5 puntos, donde 1 es muy mala y 5 muy buena.

Las preguntas que miden violencia escolar, ya sea como víctima, victimario u observador de violencia (62 en total), cuentan con las siguientes alternativas de respuesta: Todos los días, Al menos 1 vez por semana, Al menos 1 vez por mes, 2 o 3 veces al año y Nunca. Para medir la frecuencia de la violencia se utilizó una escala tipo Likert de 1 a 5 puntos, donde 5 es todos los dias y 1 es nunca.

Para realizar los análisis, el instrumento usado fue sometido a un análisis factorial exploratorio con un método de ejes principales con rotación oblicua (PROMAX). Se optó por esta rotación ya que supone correlación entre los factores, considerando que se esperaba que hubiera una asociación entre ser víctima, victimario y observador de la violencia. Según los datos nacionales, hay una asociación entre los roles que se asumen en las dinámicas de violencia escolar (Chile, Ministerio del Interior \& Adimark, 2008), lo cual comprueba la tesis de que la relación entre los factores es algo dinámico más que estático (Espelage \& Swearer, 2003). De acuerdo al gráfico de sedimentación, se optó por una solución de siete factores, los cuales dan cuenta del $43,9 \%$ de la varianza explicada del constructo usado para medir violencia escolar. Los factores obtenidos agrupan los ítems de acuerdo a distintos aspectos de la violencia escolar, a nivel de observador, agresor y víctima de violencia. A continuación se describen las escalas del instrumento que se utilizaron en el estudio.

Victimario violencia de conductas de violencia grave. Esta escala está compuesta por ocho preguntas que reportan conductas de violencia grave hacia pares o hacia docentes por parte de los alumnos. Específicamente, da cuenta del reporte que hacen los estudiantes de ser victimarios (agresores) en distintos niveles de intensidad y gravedad en conductas graves, como portar armas, robar o consumir drogas en el mismo establecimiento (e.g., ¿Has llevado armas al liceo/escuela [cuchillos, punzones, etc.]?) y haber amenazado o agredido a un docente (e.g., ¿Has insultado o agredido verbalmente a un profesor?). El nivel de consistencia interna de esta escala, estimado con alfa de Cronbach, fue 0,86 (año 2006) y 0,89 (año 2008).

Observador violencia entre pares. Esta escala está compuesta por ocho preguntas que evalúan la percepción de conductas de violencia entre pares. Específicamente, reporta la percepción de agresiones de distintos tipo (desde psicológicas hasta físicas) entre los alumnos (e.g., ¿Qué tan frecuente se da que un alumno insulte a otro dentro del liceo/escuela?). La consistencia interna de esta escala fue 0,81 (año 2006) y 0,86 (año 2008).

Víctima violencia entre pares. Esta escala está compuesta por nueve preguntas que reportan conductas de violencia recibidas por parte de pares. Específicamente, se refiere al reporte que hacen los estudiantes sobre aquellas situaciones en que son víctimas de agresión de algún tipo, con determinada intensidad, dentro del establecimiento por parte de un compañero. Dicha agresión va desde molestar o amenazar hasta agredir físicamente (e.g., Señala si dentro del liceo/escuela otros alumnos te han amenazado con hacerte daño). La consistencia interna de esta escala fue 0,84 en ambos años.

Observador violencia antisocial. Esta escala está compuesta por ocho preguntas que dan cuenta de la percepción sobre conductas de violencia entre pares y entre docentes (e.g., 
¿Con qué frecuencia se da que los alumnos peleen con armas dentro del establecimiento?, ¿Con qué frecuencia se que los adultos de tu liceo/escuela se empujen entre ellos con la intención de hacer daño?). La consistencia interna de esta escala fue 0,81 (año 2006) y 0,79 (año 2008).

Observador y víctima violencia profesores a alumnos. Esta escala incluye nueve preguntas que reportan situaciones de violencia de profesores a alumnos, tanto como víctima (e.g., Señala si un profesor te amenazó con hacerte daño) o como observador (e.g., ¿Con qué frecuencia se da que los profesores insulten a alumnos?). La consistencia interna de esta escala fue 0,88 (año 2006) y 0,87 (año 2008).

Victimario violencia compañeros. Esta escala contiene nueve preguntas que reportan violencia por parte de los alumnos hacia sus pares y el colegio; por ejemplo, haber excluido, molestado o golpeado a otro compañero (e.g., ¿Has insultado a otro alumno dentro del liceo/escuela?). La consistencia interna de esta escala fue 0,84 (año 2006) y 0,85 (año 2008).

Observador violencia alumnos a adultos. Esta escala contiene cuatro preguntas que describen conductas observadas de agresión de alumnos dirigidas a los adultos del colegio (e.g., que los alumnos empujen a los profesores con la intención de hacerle daño). La consistencia interna de esta escala fue 0,78 (año 2006) y 0,76 (año 2008).

Calidad general de la relación. Esta escala está compuesta por siete preguntas que dan cuenta de la evaluación que hacen los estudiantes acerca de las relaciones interpersonales al interior del establecimiento entre alumnos, profesores, paradocentes y apoderados, valorando su calidad (e.g., En tu escuela o liceo: ¿la relación entre los alumnos es?; ¿la relación entre los alumnos y los profesores es?; ¿la relación entre los adultos del colegio [profesor, director, inspector, personal de aseo, etc.] es?). La consistencia interna de esta escala fue 0,68 (año 2006) y 0,70 (año 2008).

\section{Procedimiento}

El cuestionario se aplicó antes de la implementación del programa (julio 2006) y durante el mes de diciembre de 2008, dos años después de la implementación del mismo. El proceso de recolección de datos fue responsabilidad de cada colegio, quienes autorizaron y coordinaron el levantamiento de la información, velando por la confidencialidad de los alumnos y el uso responsable de esta. Tanto los directores de las escuelas como el Departamento de Educación Municipal de la comuna autorizaron la aplicación del instrumento. En cada establecimiento se aplicó el instrumento en la misma sala durante el horario escolar, administrado por un educador del colegio responsable del programa, capacitado y supervisado por el coordinador del programa en la comuna. Los cuestionarios fueron anónimos y de carácter voluntario, dando plena libertad para responder o no los mismos. Una vez completados, fueron entregados al investigador, para preservar la confidencialidad de los datos.

\section{Análisis de la Información}

En una primera etapa se llevaron a cabo análisis descriptivos para explorar la distribución de los puntajes y detectar errores de codificación. En una segunda etapa se realizaron las comparaciones entre la evaluación previa (2006) y el seguimiento (2008), incluyendo todos los niveles evaluados. Para evaluar las hipótesis del estudio se compararon los promedios de cada factor antes y después de la implementación del programa, por medio de la prueba $t$ de Student para muestras dependientes. En este análisis y en los posteriores no se incluyeron los ítems del instrumento que pesaron en más de un factor. En la segunda comparación, que tuvo como objetivo identificar si hubo diferencias en los mismos cursos al cabo de dos años, se compararon las tasas de violencia de dos niveles agrupados: $5^{\circ} / 6^{\circ}$ básicos en 2006 y $7^{\circ} / 8^{\circ}$ básicos en 2008 . Antes 
de realizar todos los análisis se llevó a cabo la prueba de Levene para evaluar la homogeneidad de las varianzas. Los análisis se realizaron con el programa SPSS versión 15.0.

\section{Resultados}

La comparación de resultados se hizo en base a las tasas de violencia para cada uno de los factores obtenidos en el estudio. En la Tabla 1 se muestran los valores para cada factor, según el curso, en el año 2006, antes de implementarse el programa.

Tabla 1

Medias en Cada Factor, Según Curso, Antes de la Implementación del Programa

\begin{tabular}{|c|c|c|c|c|c|c|c|c|c|c|}
\hline \multirow[b]{2}{*}{ Rol } & \multirow[b]{2}{*}{ Factor } & \multicolumn{9}{|c|}{ Curso } \\
\hline & & Total & $\begin{array}{c}5^{\circ} \\
\text { básico }\end{array}$ & $\begin{array}{c}6^{\circ} \\
\text { básico }\end{array}$ & $\begin{array}{l}7^{\circ} \\
\text { básico }\end{array}$ & $\begin{array}{l}8^{\circ} \\
\text { básico }\end{array}$ & $\begin{array}{c}1^{\circ} \\
\text { medio }\end{array}$ & $\begin{array}{c}2^{\circ} \\
\text { medio }\end{array}$ & $\begin{array}{c}3^{\circ} \\
\text { medio }\end{array}$ & $\begin{array}{c}4^{\mathrm{o}} \\
\text { medio }\end{array}$ \\
\hline \multirow[t]{2}{*}{ Víctima } & $\begin{array}{l}\text { Víctima violencia } \\
\text { entre pares }\end{array}$ & $\begin{array}{c}2,66 \\
(1,00)\end{array}$ & $\begin{array}{c}2,69 \\
(0,96)\end{array}$ & $\begin{array}{c}2,91 \\
(1,15)\end{array}$ & $\begin{array}{c}2,70 \\
(0,94)\end{array}$ & $\begin{array}{c}2,92 \\
(0,92)\end{array}$ & $\begin{array}{c}2,27 \\
(0,88)\end{array}$ & $\begin{array}{c}2,11 \\
(0.88)\end{array}$ & $\begin{array}{c}2,23 \\
(0.93)\end{array}$ & $\begin{array}{c}2,53 \\
(0,89)\end{array}$ \\
\hline & $\begin{array}{l}\text { Observador y víctima } \\
\text { violencia profesores a } \\
\text { alumnos }\end{array}$ & $\begin{array}{c}1,51 \\
(0,78)\end{array}$ & $\begin{array}{c}1,44 \\
(0,72)\end{array}$ & $\begin{array}{c}1,47 \\
(0,76)\end{array}$ & $\begin{array}{c}1,57 \\
(0,90)\end{array}$ & $\begin{array}{c}1,58 \\
(0,80)\end{array}$ & $\begin{array}{c}1,29 \\
(0,43)\end{array}$ & $\begin{array}{c}1,50 \\
(0,78)\end{array}$ & $\begin{array}{c}1,62 \\
(087)\end{array}$ & $\begin{array}{c}1,61 \\
(0,62)\end{array}$ \\
\hline \multirow[t]{3}{*}{ Testigo } & $\begin{array}{l}\text { Observador violencia } \\
\text { alumnos a adultos }\end{array}$ & $\begin{array}{c}2,50 \\
(1,05)\end{array}$ & $\begin{array}{c}2,29 \\
(1,08)\end{array}$ & $\begin{array}{c}2,52 \\
(1,17)\end{array}$ & $\begin{array}{c}2,35 \\
(0,96)\end{array}$ & $\begin{array}{c}2,63 \\
(0,95)\end{array}$ & $\begin{array}{l}2,45 \\
(0,95)\end{array}$ & $\begin{array}{c}2,51 \\
(1,01)\end{array}$ & $\begin{array}{c}2,98 \\
(1,06)\end{array}$ & $\begin{array}{c}3,03 \\
(1,07)\end{array}$ \\
\hline & $\begin{array}{l}\text { Observador violencia } \\
\text { antisocial }\end{array}$ & $\begin{array}{c}1,81 \\
(0,81)\end{array}$ & $\begin{array}{c}1,54 \\
(0,80)\end{array}$ & $\begin{array}{c}1,72 \\
(0,75)\end{array}$ & $\begin{array}{c}1,72 \\
(0,82)\end{array}$ & $\begin{array}{c}1,98 \\
(0,86)\end{array}$ & $\begin{array}{c}1,89 \\
(0,52)\end{array}$ & $\begin{array}{c}2,04 \\
(0,81)\end{array}$ & $\begin{array}{c}1,89 \\
(0,61)\end{array}$ & $\begin{array}{c}2,36 \\
(0,90)\end{array}$ \\
\hline & $\begin{array}{l}\text { Observador violencia } \\
\text { entre pares }\end{array}$ & $\begin{array}{c}4,08 \\
(0,78)\end{array}$ & $\begin{array}{c}3,94 \\
(0,90)\end{array}$ & $\begin{array}{c}4,16 \\
(0,83)\end{array}$ & $\begin{array}{c}4,08 \\
(0,83)\end{array}$ & $\begin{array}{c}4,15 \\
(0,67)\end{array}$ & $\begin{array}{c}4,10 \\
(0,59)\end{array}$ & $\begin{array}{c}3,96 \\
(0,77)\end{array}$ & $\begin{array}{c}4,15 \\
(0,54)\end{array}$ & $\begin{array}{c}4,00 \\
(0,66)\end{array}$ \\
\hline \multirow[t]{2}{*}{ Victimario } & $\begin{array}{l}\text { Victimario violencia } \\
\text { de conductas de } \\
\text { violencia grave }\end{array}$ & $\begin{array}{c}1,27 \\
(0,59)\end{array}$ & $\begin{array}{c}1,24 \\
(0,60)\end{array}$ & $\begin{array}{c}1,24 \\
(0,51)\end{array}$ & $\begin{array}{c}1,23 \\
(0,52)\end{array}$ & $\begin{array}{c}1,41 \\
(0,82)\end{array}$ & $\begin{array}{l}1,24 \\
(0,57)\end{array}$ & $\begin{array}{l}1,20 \\
(0,41)\end{array}$ & $\begin{array}{c}1,22 \\
(0,36)\end{array}$ & $\begin{array}{c}1,31 \\
(0,47)\end{array}$ \\
\hline & $\begin{array}{l}\text { Victimario violencia } \\
\text { compañeros }\end{array}$ & $\begin{array}{c}2,20 \\
(0,92)\end{array}$ & $\begin{array}{c}2,06 \\
(0,92)\end{array}$ & $\begin{array}{c}2,22 \\
(0,91)\end{array}$ & $\begin{array}{c}2,16 \\
(0,89)\end{array}$ & $\begin{array}{c}2,43 \\
(0,99)\end{array}$ & $\begin{array}{c}2,13 \\
(0,71)\end{array}$ & $\begin{array}{c}1,98 \\
(0,88)\end{array}$ & $\begin{array}{c}2,16 \\
(0,94)\end{array}$ & $\begin{array}{c}2,43 \\
(0,99)\end{array}$ \\
\hline Clima & $\begin{array}{l}\text { Calidad general de la } \\
\text { relación }\end{array}$ & $\begin{array}{c}3,64 \\
(0,54)\end{array}$ & $\begin{array}{c}3,77 \\
(0,62)\end{array}$ & $\begin{array}{c}3,58 \\
(0,55)\end{array}$ & $\begin{array}{c}3,63 \\
(0,57)\end{array}$ & $\begin{array}{c}3,63 \\
(0,46)\end{array}$ & $\begin{array}{c}3,72 \\
(0,54)\end{array}$ & $\begin{array}{c}3,71 \\
(0,45)\end{array}$ & $\begin{array}{c}3,36 \\
(0,53)\end{array}$ & $\begin{array}{c}3,52 \\
(0,39)\end{array}$ \\
\hline
\end{tabular}

Nota. Las desviaciones estándar aparecen entre paréntesis.

En la Tabla 2 se reportan los resultados de la comparación de las tasas de violencia de los factores antes (2006) y después (2008) de la implementación del programa en los mismos cursos (por ejemplo, $6^{\circ}$ en 2006 y $6^{\circ}$ en 2008). Los resultados de la prueba de Levene aconsejaron considerar las varianzas distintas en el caso de los factores Observador y Víctima Violencia Profesores a Alumnos, Levene $(1,911)=7,71, p=0,006$, y Observador Violencia Antisocial, Levene $(1,911)=6,64, p=0,011$. Se reportan los resultados ajustados considerando los resultados de esta prueba.

Como se puede apreciar en la Tabla 2, hay una reducción en el reporte de agresión recibida como víctimas en sus dos factores, al igual que en los cuatro factores que evalúan la percepción de violencia. Sin embargo, el tamaño del efecto es pequeño, según Cohen (1992). En relación con el reporte de agresores, solo se observó una reducción del reporte en el factor Victimario Violencia Compañeros pero no en el factor Victimario Violencia de Conductas de Violencia Grave. También se apreció un aumento en el factor Calidad General de la Relación. 
Tabla 2

Comparación de las Medias por Factor Entre los Años 2006 y 2008

\begin{tabular}{|c|c|c|c|c|c|c|c|}
\hline \multirow{2}{*}{ Rol } & \multirow{2}{*}{ Factor } & \multicolumn{2}{|c|}{ Media $(D E)$} & \multirow{2}{*}{$\begin{array}{c}95 \% \text { IC } \\
\text { diferencia de } \\
\text { las medias }\end{array}$} & \multirow{2}{*}{$t(p)$} & \multirow{2}{*}{$g l$} & \multirow{2}{*}{$\begin{array}{l}d \text { de } \\
\text { Cohen }\end{array}$} \\
\hline & & 2006 & 2008 & & & & \\
\hline \multirow[t]{2}{*}{ Víctima } & $\begin{array}{l}\text { Víctima violencia entre } \\
\text { pares }\end{array}$ & $2,66(1,00)$ & $2,48(0,55)$ & {$[-0,11,-0,27]$} & $5,79(<0,01)$ & 915 & 0,19 \\
\hline & $\begin{array}{l}\text { Observador y víctima } \\
\text { violencia profesores a } \\
\text { alumnos }\end{array}$ & $1,51(0,78)$ & $1,38(0,19)$ & {$[-0,05,-0,21]$} & $3,92(<0,01)$ & 911 & 0,18 \\
\hline \multirow[t]{3}{*}{ Testigo } & $\begin{array}{l}\text { Observador violencia } \\
\text { alumnos a adultos }\end{array}$ & $2,50(1,05)$ & $2,16(0,85)$ & {$[-0,26,-0,44]$} & $12,22(<0,01)$ & 917 & 0,34 \\
\hline & $\begin{array}{l}\text { Observador violencia } \\
\text { antisocial }\end{array}$ & $1,81(0,81)$ & $1,65(0,41)$ & {$[-0,11,-0,23]$} & $6,56(<0,01)$ & 911 & 0,22 \\
\hline & $\begin{array}{l}\text { Observador violencia } \\
\text { entre pares }\end{array}$ & $4,08(0,78)$ & $3,91(0,48)$ & {$[-0,10,-0,24]$} & $6,14(<0,01)$ & 913 & 0,21 \\
\hline \multirow[t]{2}{*}{ Victimario } & $\begin{array}{l}\text { Victimario violencia de } \\
\text { conductas de violencia } \\
\text { grave }\end{array}$ & $1,27(0,59)$ & $1,24(0,09)$ & & $1,77 \quad(0,12)$ & 910 & \\
\hline & $\begin{array}{l}\text { Victimario violencia } \\
\text { compañeros }\end{array}$ & $2,20(0,92)$ & $2,06(0,49)$ & {$[-0,08,-0,21]$} & $5,42(<0,01)$ & 917 & 0,17 \\
\hline Clima & $\begin{array}{l}\text { Calidad general de la } \\
\text { relación }\end{array}$ & $3,64(0,54)$ & $3,71(0,26)$ & {$[0,01,0,10]$} & $2,95(<0,01)$ & 918 & 0,13 \\
\hline
\end{tabular}

Nota. Solo se han consignado los intervalos de confianza (IC) y $d$ de Cohen respecto de las diferencias significativas.

Al agrupar a los participantes por nivel $\left(5^{\circ} / 6^{\circ}\right.$ en 2006 y $7^{\circ} / 8^{\circ}$ en 2008$)$, los resultados presentados en la Tabla 3 muestran diferencias en las tasas de solo dos factores de violencia antes y después de la implementación del programa, específicamente, Observador y Víctima Violencia Profesores a Alumnos y Observador Violencia Alumnos a Adultos.

\section{Discusión}

El presente estudio tuvo como objetivo evaluar si el programa de intervención Recoleta en Buena fue efectivo para disminuir la violencia escolar. Específicamente, se esperaba encontrar una disminución en los reportes de violencia como víctima, agresor y observador y una mejoría en los indicadores de clima social escolar. Los resultados mostraron que en promedio los estudiantes de los colegios del estudio disminuyeron sus reportes de violencia según si eran víctimas, victimarios y testigos, a excepción del factor Victimario de Violencia de Conductas de Violencia Grave. Este último resultado apoya parcialmente las hipótesis del estudio, ya que muestra que los estudiantes de cursos equivalentes de los colegios intervenidos reportan menores niveles de violencia después de dos años, pero no es así en los reportes de victimarios de violencia y en especial en aquellas situaciones evaluadas como graves. Este resultado puede dar cuenta de la efectividad del programa en materias promocionales y preventivas (primaria, específicamente), logrando incidir a nivel de toda la institución escolar y en aquellas variables grupales. Se puede explicar porque el programa evaluado trabajó de forma universal las normas de conducta de los reglamentos escolares de los colegios, a través de la enseñanza y refuerzo de expectativas de conducta, elemento descrito como una estrategia efectiva para prevenir violencia escolar (Gottfredson, 1997; Sprague \& Walker, 2000, entre otros) y cuya influencia puede ser mayor si estas han sido definidas por todos los estamentos educacionales, como fue en este caso (Farrington \& Ttofi, 2009). 
Tabla 3

Comparación de las Medias por Factor Entre $5^{\circ} / 6^{\circ}$ en 2006 y $7^{\circ} / 8^{\circ}$ en 2008

\begin{tabular}{|c|c|c|c|c|c|c|c|}
\hline \multirow{2}{*}{ Rol } & \multirow{2}{*}{ Factor } & \multicolumn{2}{|c|}{ Media $(D E)$} & \multirow{2}{*}{$\begin{array}{c}95 \% \text { IC } \\
\text { diferencia de } \\
\text { las medias }\end{array}$} & \multirow{2}{*}{$t(p)$} & \multirow{2}{*}{$g l$} & \multirow{2}{*}{$\begin{array}{l}d \text { de } \\
\text { Cohen }\end{array}$} \\
\hline & & $5^{\circ} / 6^{\circ}$ en 2006 & $7^{\circ} / 8^{\circ}$ en 2008 & & & & \\
\hline \multirow[t]{2}{*}{ Víctima } & $\begin{array}{l}\text { Víctima violencia entre } \\
\text { pares }\end{array}$ & $2,81(0,43)$ & $2,65(0,59)$ & & $2,05(0,07)$ & 434 & \\
\hline & $\begin{array}{l}\text { Observador y víctima } \\
\text { violencia profesores a } \\
\text { alumnos }\end{array}$ & $1,46(0,23)$ & $1,35(0,16)$ & {$[-0,02,-0,18]$} & $2,93(0,01)$ & 439 & 0,14 \\
\hline \multirow[t]{3}{*}{ Testigo } & $\begin{array}{l}\text { Observador violencia } \\
\text { alumnos a adultos }\end{array}$ & $2,42(0,84)$ & $2,25(0,87)$ & {$[-0,04,-0,37]$} & $2,50(0,01)$ & 439 & 0,15 \\
\hline & $\begin{array}{l}\text { Observador violencia } \\
\text { antisocial }\end{array}$ & $1,64(0,24)$ & $1,65(0,41)$ & & $0,18(0,86)$ & 431 & \\
\hline & $\begin{array}{l}\text { Observador violencia } \\
\text { entre pares }\end{array}$ & $4,06(0,34)$ & $4,05(0,42)$ & & $0,21(0,84)$ & 433 & \\
\hline \multirow[t]{2}{*}{ Victimario } & $\begin{array}{l}\text { Victimario violencia de } \\
\text { conductas de violencia } \\
\text { grave }\end{array}$ & $1,24(0,10)$ & $1,26(0,09)$ & , & $1,27(0,24)$ & 396 & \\
\hline & $\begin{array}{l}\text { Victimario violencia } \\
\text { compañeros }\end{array}$ & $2,15(0,45)$ & $2,13(0,56)$ & & $0,18(0,86)$ & 435 & \\
\hline Clima & $\begin{array}{l}\text { Calidad general de la } \\
\text { relación }\end{array}$ & $3,67(0,28)$ & $3,66(0,28)$ & & $0,20(0,85)$ & 439 & \\
\hline
\end{tabular}

Nota. Solo se han consignado los intervalos de confianza (IC) y $d$ de Cohen respecto de las diferencias significativas.

Por otro lado, la participación de los padres y apoderados en este proceso es un factor que puede haber influido en los resultados. Estudios anteriores han mostrado que aquellas estrategias de intervención que incorporan también a los padres y apoderados, dado que permiten sensibilizarlos respecto a esta problemática y facilitan un trabajo colaborativo entre apoderados y profesores, también permiten predecir un mejor resultado en los programas de intervención, porque se asume un desafío que considera a toda la comunidad escolar (Ortega et al., 2003).

Al comparar la violencia en los mismos niveles entre el año 2006 y 2008, los resultados sugieren que este programa influye menos en la reducción de la violencia escolar cuando ya está instalada como patrón de conducta en niños, niñas y adolescentes. Este resultado es consistente con los de estudios anteriores que sugieren que las estrategias de trabajo individual con víctimas y victimarios, como se implementó en este caso para intervenir en estas conductas, son menos eficaces en la reducción de la violencia escolar (Farrington \& Ttofi, 2009). Los investigadores proponen que, a pesar de estos resultados, son necesarios esfuerzos orientados a desarrollar estrategias efectivas de intervención con victimarios, para lograr cambios en la conducta (Losel \& Beelman, 2003). Por lo tanto, sería necesario implementar intervenciones más complejas/específicas o de nivel terciario para trabajar con este grupo, más allá de las desarrolladas como parte de este programa.

El programa evaluado pareciera no influir en los reportes de victimarios de violencia más grave o delictual. Si bien no hubo un aumento en esta dimensión, el hecho de que no haya disminuido hace necesario implementar metodologías de trabajo aún más focalizadas. La no disminución puede ser explicada por una naturalización o validación general de este tipo de violencia escolar en los contextos de las escuelas evaluadas. Tal como se describió, el programa desarrollaba estrategias en distintos niveles de intervención, pero no fue capaz de dar un apoyo sistemático y focalizado a alumnos en mayor situación de riesgo (derivación a redes de apoyo sociales de la comuna), considerando los altos niveles de vulnerabilidad de los colegios. Esto da cuenta de la importancia de trabajar no solo con variables escolares (normas de conductas), sino también con variables psicosociales que pueden incidir en la manifestación del fenómeno al interior del establecimiento, acorde con una perspectiva más ecológica. Podría pensarse, sin 
embargo, que no debería esperarse que un programa de esta naturaleza, el cual fortalece las relaciones interpersonales (positive support), incida en conductas de tipo delictuales, ya que su intervención requiere de otro tipo de metodología y actores. Es posible que otros enfoques puedan ser más efectivos en casos más severos de violencia, como, por ejemplo, enfoques basados en la teoría de la resistencia, en los cuales se trabaja la relación con los victimarios y las formas en que se implementan las sanciones a las conductas violentas (Farrington \& Ttofi, 2009).

Es importante señalar que, si bien los puntajes en todas las escalas de violencia no disminuyeron, tampoco aumentaron en la segunda medición. Aun cuando el programa tuvo como objetivo reducir la violencia en las escuelas intervenidas, este resultado puede ser alentador si se comparan estas estadísticas con las nacionales, que sugieren que la curva de violencia escolar es mayor en los niveles finales del segundo ciclo de enseñanza básica, es decir, $7^{\circ}$ y $8^{\circ}$ básicos (Chile, Ministerio del Interior \& Adimark, 2008). Estos resultados pueden explicarse como producto del desarrollo de estrategias de prevención secundaria y terciaria del programa evaluado (enseñanza de habilidades sociales, tutorías y apoyos focalizados en cursos problemáticos), las cuales intervienen en manifestaciones de la violencia. De acuerdo a esto, sería interesante poder realizar en el futuro un seguimiento de las mismas escuelas evaluadas para ver la mantención de los resultados.

El estudio realizado presenta limitaciones que son importantes de señalar, especialmente en el diseño. Dado que no contó con un grupo control y los grupos antes y después de la intervención no fueron equivalentes, se hace difícil atribuir al programa el logro en la disminución de la violencia. Las estadísticas de los estudios nacionales sugieren que la violencia escolar en Chile ha disminuido, excepto en los actos de agresión más graves (agresiones sexuales, robo, porte de armas), los que han ido en aumento (Chile, Ministerio del Interior \& Adimark, 2008), lo cual también puede explicar los resultados encontrados en este estudio. Además, los instrumentos utilizados fueron de carácter anónimo, lo cual no permitió llevar a cabo comparaciones entre grupos equivalentes o a nivel individual. Sin embargo, usar esta metodología en la evaluación de programas no ha sido una limitante en otras partes del mundo. Por ejemplo, en España, aunque se ha visto un descenso de la violencia escolar en el último tiempo (Gázquez, Pérez-Fuentes, Carrión \& Santiuste, 2010), se reconocen los impactos del programa de Rosario Ortega cuando ha sido implementado y evaluado con una metodología similar (Ortega et al., 2003).

Otra limitación del estudio fue la participación de profesores de los colegios como ayudantes en el levantamiento de información, lo cual podría haber incidido en las respuestas de los alumnos. Esto se relaciona con otra limitación ética importante del estudio, ya que, si bien se contó con el permiso de los colegios y el instrumento era anónimo y voluntario para los alumnos, no se contó con la autorización directa de los padres y apoderados. Esto se entiende a la luz de la inexperiencia en evaluación de programas de parte del equipo. Por la misma razón, no se consideró un grupo control ni se hizo un seguimiento focalizado de los mismos individuos.

Si bien la comparación realizada fue un pre-test y post-test con dos años de diferencia, fue llevada a cabo en meses diferentes (julio del 2006 y diciembre del 2008), debido a la disponibilidad de recursos de los ejecutores. Esto puede ser una limitación adicional del estudio, porque es posible que los valores reportados de violencia varíen a lo largo del año. Esto fue descrito en Chile en un estudio que buscaba comparar la frecuencia e intensidad de las interacciones agresivas de alumnos y alumnas durante diferentes estaciones del año, para establecer la posible ocurrencia de cambios estacionales en la dinámica de la violencia. En sus resultados se evidenció que había un incremento de la frecuencia de interacciones agresivas durante el período primavera-verano, comparado con el período otoño-invierno. Sin embargo, este estudio se realizó solo en un establecimiento educacional (Beltrami, Sanhueza, Higueras, Flores \& Muñoz, 2007). Otros estudios de la región también han encontrado diferencias en el reporte de agresión a lo largo del año escolar. Específicamente, Saldarriaga, Bukowski y Velásquez (2010) evidenciaron un descenso de los reportes de victimización de pre-adolescentes colombianos a lo largo del año, en particular en aquellos que presentaban niveles más altos al comienzo del año. En este sentido, es importante tener presente que los resultados del programa pueden haber respondido a variables ajenas al mismo. 
A pesar de estas limitaciones, el presente estudio entrega información sobre la evaluación de la efectividad de una estrategia de intervención en violencia escolar en una comuna del país. Como se señaló, en Chile actualmente existe escasa evaluación e información respecto de este tema y estudios de esta naturaleza pueden ayudan a contribuir a la implementación de estrategias efectivas de intervención de esta problemática.

En relación con los estándares de evaluación antes descritos —Escala de Maryland-, el presente estudio de evaluación correspondería a un valor de dos, en una escala de 1 a 5 (Grove, 2010). Sin embargo, al no haber controlado elementos externos al programa, no es posible hablar de efectividad de la intervención. Esto muestra las brechas en la evaluación de estrategias de intervención en prevención, incluso en campos diferentes a la violencia escolar. Los programas no están pensados desde su formulación inicial en incluir robustos sistemas de evaluación, lo cual también podría explicar el hecho de que en esta investigación no se haya solicitado la autorización de los apoderados para las evaluaciones y solo se haya solicitado la de los colegios. Estos elementos deberían alimentar las políticas de prevención en programas de esta naturaleza, agregando componentes de evaluación con estándares de mayor nivel, tales como tamaño del efecto y sostenibilidad en el tiempo, entre otros (Olweus, 2004).

El estudio realizado tomó en su conjunto a los cuatro establecimientos que participaron del programa. Sin embargo, no se hizo un análisis por separado de cada uno de estos, lo cual podría considerarse a futuro, para identificar variables más específicas en cada colegio que pudieran mostrar aspectos exitosos. Por lo mismo, se recomienda a futuro seguir profundizando en evaluaciones de programas en Chile, utilizando diseños mixtos y meta-análisis, lo cual permitiría perfeccionar las estrategias de intervención, incidir en la realidad nacional e impactar en las políticas públicas relacionadas con el tema. Finalmente, el estudio no estableció diferencias de género ni de edad, lo cual podría ser considerado en nuevos estudios en la materia para ver con mayor detalle logros y desafíos según estas dos variables.

\section{Referencias}

Ajenjo, F. \& Bas, J. (2005). Diagnóstico de violencia escolar. Manuscrito no publicado, Pontificia Universidad Católica de Chile \& Fundación Paz Ciudadana, Santiago, Chile.

Araos, C. \& Correa, V. (2004). La escuela hace la diferencia. Aproximación sociológica a la violencia escolar (Tesis de Grado no publicada), Instituto de Sociología, Pontificia Universidad Católica de Chile \& Fundación Paz Ciudadana, Santiago, Chile.

Arón, A. M. (2000). Un programa de educación para la no violencia. Psykhe, 9(2), 25-39.

Arón, A. M. \& Milicic, N. (1999). Clima social escolar y desarrollo personal: un programa de mejoramiento. Santiago, Chile: Editorial Andrés Bello.

Arón, A. M. \& Milicic, N. (2000). Climas sociales tóxicos y climas nutritivos para el desarrollo personal en el contexto escolar. Psykhe, 9(2), 117-124.

Beltrami, M., Sanhueza, S., Higueras, T., Flores, L. \& Muñoz, J. (2007). Análisis comparativo estacional de la conducta agresiva de escolares de un establecimiento educacional de la Región Metropolitana, Santiago de Chile. Revista Chilena de Educación Científica, 6(2), 55-61.

Berger, C., Karimpour, R. \& Rodkin, P. C. (2008). Bullies and victims at school: Perspectives and strategies for primary prevention. En T. W. Miller (Ed.), School violence and primary prevention (pp. 295-322). New York, NY: Springer.

Berger, C. \& Lisboa, C. (2008). Hacia una comprensión ecológica de la agresividad entre pares en el microsistema escolar. En C. Berger \& C. Lisboa (Eds.), Violencia escolar: estudios y posibilidades de intervención en Latinoamérica (pp. 59-81). Santiago, Chile: Editorial Universitaria.

Bronfenbrenner, U. (1987). La ecología del desarrollo humano. Experimentos en entornos naturales y diseñados. Barcelona, España: Paidós.

Casassus, J. (2003). La escuela y la (des) igualdad. Santiago, Chile: LOM.

Chaux, E. (2005). El programa de prevención de Montreal: lecciones para Colombia. Revista de Estudios Sociales de la Universidad de Los Andes, 21, 11-25.

Chile, Ministerio del Interior \& Adimark (2008). Encuesta de violencia en el ámbito escolar 2005-2007. Santiago, Chile: Autores.

Cohen, J. (1992). A power primer. Psychological Bulletin, 112, 155-159. doi:10.1037/0033-2909.112.1.155

Cohen, E. \& Franco, R. (1992). Evaluación de proyectos sociales. Ciudad de México, México: Siglo XXI.

Contador, M. (2001). Percepción de la violencia escolar en estudiantes de enseñanza media. Psykhe, 10(1), 69-80.

Espelage, D. L. \& Swearer, S. M. (2003). Research on school bullying and victimization: What have we learned and where do we go from here? School Psychology Review, 32, 365-379. 
Farrington, D. P. (1993). Understanding and preventing bullying. Crime and Justice, 17, 381-458. doi:10.1086/449217 Farrington, D. P. \& Ttofi, M. M. (2009). School-based programs to reduce bullying and victimization (Campbell Systematic Reviews 2009:6). Oslo, Noruega: The Campbell Colaboration. doi:10.4073/csr.2009.6

García, M. \& Madriaza, P. (2005). Sentido y sinsentido de la violencia escolar: análisis cualitativo del discurso de estudiantes chilenos. Psykhe, 14(1), 165-180. doi:10.4067/S0718-22282005000100013

Gázquez, J. J., Pérez-Fuentes, M. C., Carrión, J. J. \& Santiuste, V. (2010). Estudio y análisis de conductas violentas en educación secundaria en España. Universitas Psychologica, 9, 371-380.

Gottfredson, D. C. (1997). School-based crime prevention. En L. W. Sherman, D. Gottfredson, D. MacKenzie, J. Eck, P. Reuter \& S. Bushway (Eds.), Preventing crime. What works, what doesn't, what's promising: A report to the United States Congress (pp. 224-249). Washington, DC: US Department of Justice, National Institute of Justice.

Gottfredson, D. C., Wilson, D. B. \& Skroban, S. (2002). School-based crime prevention. En L. W. Sherman, D. P. Farrington, B. C. Welsh \& D. L. MacKenzie (Eds.), Evidence-based crime prevention (pp. 56-164). London, Reino Unido: Routledge.

Grove, L. E. (2010). Synergies of syntheses: A comparison of systematic review and scientific realist evaluation methods for crime prevention (Tesis de Doctorado no publicada), Loughborough University, Leicestershire, Reino Unido. Extraído de https://dspace.lboro.ac.uk/dspace-jspui/bitstream/2134/6756/2/LG\%20thesis\%20final\%20version\%20f or\%20IJC.pdf

Hernández, R., Fernández, C. \& Baptista, P. (1991). Metodología de la investigación. Ciudad de México, México: Mcgraw Hill.

Krug, E. G., Dalhberg, L. L., Mercy, J. A., Zwi, A. B. \& Lozano, R. (2003). World report on violence and health. Genève, Suiza: World Health Organization. Extraído de http://www.who.int/violence_injury_prevention/violence/world_ report/wrvh1/en/

Lösel, F. \& Beelmann, A. (2003). Effects of child skills training on preventing antisocial behavior: A systematic review of randomized evaluations. The Annals of the American Academy of Political and Social Science, 587, 84-109. doi:10.1177/0002716202250793

Olweus, D. (2004). The Olweus Bullying Prevention Program: Design and implementation issues and a new national initiative in Norway. En P. K. Smith, D. Pepler \& K. Rigby (Eds.), Bullying in schools: How successful can interventions be? (pp. 13-36). Cambridge, Reino Unido: Cambridge University Press.

Orpinas, P. \& Horne, A. M. (2006). Bullying prevention: Creating a positive school climate and developing social competence. Washington, DC: American Psychological Association. doi:10.1037/11330-000

Ortega, R., Del Rey, R. \& Fernández, I. (2003). Working together to prevent school violence: The Spanish response. En P. K. Smith (Ed.), Violence in schools: The response in Europe (pp. 209-240). London, Reino Unido: Routledge.

OSEP Center on Positive Behavioral Interventions, Sugai, G., Horner, R. H., Dunlap, G., Hieneman, M., Lewis, T. J. ... Ruef, M. (2000). Applying positive behavior support and functional behavioral assessment in schools. Journal of Positive Behavior Interventions, 2, 131-143. doi:10.1177/109830070000200302

Pérez, V., Rodríguez, J., De la Barra, F. \& Fernández, A. M. (2005). Efectividad de una estrategia conductual para el manejo de la agresividad en escolares de enseñanza básica. Psykhe, 14(2), 55-62. doi:10.4067/S071822282005000200005

Przybylski, R. (2008). What works, effective recidivism reduction and risk-focused prevention programs: A compendium of evidence-based options for preventing new and persistent criminal behavior. Denver, CO: Colorado Division of Criminal Justice, Department of Public Safety.

Rigby, K. (2003). Addressing bullying in schools: Theory and practice (Trends \& Issues in Crime and Criminal Justice $\mathrm{N}^{\circ}$ 259). Canberra, Australia: Australian Institute of Criminology.

Rigby, K. (2004). Addressing bullying in schools: Theoretical perspectives and their implications. School Psychology International, 25, 287-300. doi:10.1177/0143034304046902

Rigby, K. (2005). Why do some children bully at school? The contributions of negative attitudes towards victims and the perceived expectations of friends, parents and teachers. School Psychology International, 26, 147-161. doi:10 $.1177 / 0143034305052910$

Rigby, K., Smith, P. \& Pepler, D. (2004). Working to prevent school bullying: Key issues. En P. K. Smith, D. Pepler \& K. Rigby (Eds.), Bullying in schools: How successful can interventions be? (pp. 1-12). Cambridge, Reino Unido: Cambridge University Press.

Saldarriaga, L. M., Bukowski, W. \& Velásquez, A. M. (2010). The moderating effects of prosocial behaviour in the relationship between risk factors and peer victimization in Colombian early adolescents (Tesis de Doctorado no publicada), Concordia University, Montreal, Canadá.

Schneider, T., Walker, H. \& Sprague, J. (2000). Safe school design: A handbook for educational leaders applying the principles of crime prevention through environmental design. Eugene, OR: ERIC Clearinghouse on Educational Management.

Shadish, W. R., Cook, T. D. \& Campbell, D. T. (2002). Experimental and quasi-experimental designs for generalized causal inference. Boston, MA: Houghton Mifflin.

Sherman, L. W., Gottfredson, D., MacKenzie, D., Eck, J., Reuter, P. \& Bushway, S. (1997). Preventing crime. What works, what doesn't, what's promising: A report to the United States Congress. Washington, DC: US Department of Justice, National Institute of Justice.

Sprague, J. R. \& Golly, A. (2005). Best behavior: Building positive behavior support in schools. Frederick, CO: Sopris West Educational Services.

Sprague, J. R. \& Walker, H. M. (2000). Early identification and intervention for youth with antisocial and violent behavior. Exceptional Children, 66, 367-379. 
Sprague, J. R. \& Walker, H. M. (2005). Safe and healthy schools: Practical prevention strategies. New York, NY: The Guilford Press.

Sugai, G. (2003). Commentary: Establishing efficient and durable systems of school-based support. School Psychology Review, 32, 530-535.

Sugai, G. (2007). Promoting behavioral competence in schools: A commentary on exemplary practices. Psychology in the Schools, 44, 113-118. doi:10.1002/pits.20210

Sugai, G. \& Horner, R. R. (2006). A promising approach for expanding and sustaining school-wide positive behavior support. School Psychology Review, 35, 245-259.

Tamar, F. (2005). Maltrato entre escolares (bullying): estrategias de manejo que implementan los profesores al interior del establecimiento escolar. Psykhe, 14(1), 211-225. doi:10.4067/S0718-22282005000100016

Tijmes, C. \& Varela, J. (2008, Noviembre). Resultados programa de prevención de la violencia en las escuelas "Paz Educa”. Ponencia presentada en el Quinto Congreso Nacional de Investigación sobre Violencia y Delincuencia, Fundación Paz Ciudadana/Pontificia Universidad Católica de Chile, Instituto de Sociología, Santiago, Chile.

Turnbull, A., Edmonson, H., Griggs, P., Wickham, D., Sailor W., Freeman, R. ... Warren, J. (2002). A blueprint for schoolwide positive behavior support: Implementation of three components. Exceptional Children, 68, 377-402.

Vanderschueren, F. \& Lunecke, A. (2004). Prevención de la delincuencia juvenil: análisis de experiencias internacionales. Santiago, Chile: Ministerio del Interior, División de Seguridad Ciudadana/Banco Interamericano de Desarrollo/ Universidad Alberto Hurtado.

Varela, J., Farren, D. \& Tijmes, C. (2010). Violencia escolar en educación básica: evaluación de un instrumento para su medición. Santiago, Chile: Fundación Paz Ciudadana.

Varela, J., Tijmes, C. \& Sprague, J. (2009). Paz Educa. Prevención de la violencia escolar. Santiago, Chile: Fundación Paz Ciudadana.

Welsh, B. C. (2007). Evidence-based crime prevention: Scientific basis, trends, results and implications for Canada. Otawa, Canadá: National Crime Prevention Centre. Extraído de http://www.publicsafety.gc.ca/prg/cp/_fl/ evidenced-based-cp-e.pdf

Fecha de recepción: Marzo de 2011.

Fecha de aceptación: Octubre de 2011. 